\title{
Analytic functions and linearly ordered groups*
}

by

I. I. HIRSCHMAN, Jr. (St. Louis, Mo.)

Abstract. If $\sum_{0}^{\infty}|f(n)|<\infty$ then $\left\{\theta: f^{\wedge}(\theta)=0\right\}$, where $f^{\wedge}(\theta)=\sum_{0}^{\infty} f(n) e^{i n \theta}$, has measure 0 . It is shown that if the integer group replaced by an arbitrary linearly ordered discrete group then a (weak) analogue of this result is valid.

Let $Z$ be the additive group of integers and let $L^{1}(Z)$ consist of those complex valued functions $f$ on $Z$ for which $\|f\|_{1}<\infty$ where

$$
\|f\|_{1}=\sum_{Z}|f(n)| \text {. }
$$

We say that $f \epsilon A^{1}(Z)$ if $f \epsilon L^{1}(Z)$ and if $f(n)=0$ for all $n<0$. Let $R$ be the real numbers and let $T=R / 2 \pi Z$. If $f \in A^{1}(Z), f \neq 0$, and if

$$
f^{\wedge}(\theta)=\sum_{Z} e^{i n \theta} f(n) \quad \theta \in T,
$$

then $\left\{\theta: f^{\wedge}(\theta)=0\right\}$ is a (closed) set in $T$ of measure 0. On the contrary if $f \in L^{1}(Z), f \neq 0$, then $\left\{\theta: f^{\wedge}(\theta)=0\right\}$ can be an arbitrary closed set of measure less than $2 \pi$. A weaker version of this statement, which does not depend on a detailed description of $T$ is the following. If $f \in A^{1}(Z)$, $f \neq 0$, if $g \in L^{1}(Z)$, and if

$$
\left\{\theta: g^{1}(\theta) \neq 0\right\} \subset\left\{\theta: f^{\wedge}(\theta)=0\right\},
$$

then $g \equiv 0$. We will show that properly interpreted this statement holds for an arbitrary linearly ordered discrete group $G$, which need not be Abelian.

Let $G$ be a group with elements $a, b, c, \ldots ; e$ is the identity of $G$. We assume that there has been distinguished on $G$ a linear order relation " $<$ " compatible with the group structure, that is:

(i) for each $a, b \in G$ exactly one of $a=b, a>b$ or $a<b$ holds;

(1) (ii) $a<b$ and $b<c$ implies $a<c$;

(iii) $a<b$ implies $c a<c b$ and $a c<b c$ for all $c \epsilon G$.

* Research supported by the U.S. National Science Foundation under NSF Grant GP-6907. 
We take $G$ in the discrete topology. $G$ is then unimodular and an invariant measure is obtained by assigning mass 1 to each point of $G$. $L^{1}(G)$ consists of all complex valued functions $f$ on $G$ for which $\|f\|_{1}<\infty$ where

$$
\|f\|_{1}=\sum_{G}|f(a)| .
$$

We say that $f \in A^{1}(G)$ if $f \in L^{1}(G)$ and if $f(a)=0$ for all $a<e$.

Let $\Omega$ be the set of equivalence classes of irreducible unitary representations of $G$. For each $\omega \epsilon \Omega$ we choose a representation $\left[U_{\omega}(\cdot), H_{\omega}\right]$ from $\omega$. Here $H_{\omega}$ is a Hilbert space and $a \rightarrow V_{\omega}(a)$ is a homomorphism of $G$ into an irreducible group of unitary operators on $H_{\omega}$. Given $g \in L^{1}(G)$ we set

$$
[\alpha, \beta]_{g}=\sum_{a \in G} g(a)\left\langle D_{\omega}(a) \alpha, \beta\right\rangle .
$$

Here $\alpha, \beta \epsilon H_{\omega}$, and $\langle\cdot, \cdot\rangle$ is the inner product in $H_{\omega}$. It is easily verified that

$$
\left|[\alpha, \beta]_{g}\right| \leqslant\|\alpha\|\|\beta\|\|g\|_{1} .
$$

Since $[\alpha, \beta]_{g}$ is sesquilinear it follows that there is a unique bounded 1 inea transformation $g^{\wedge}(\omega)$ on $H_{\omega}$ such that for all $a, \beta \in H_{\omega}$

$$
[\alpha, \beta]_{g}=\left\langle g^{\wedge}(\omega) \alpha, \beta\right\rangle .
$$

We can now state our principal result.

THEOREM 1. Let $G$ be as in (1), and let $g \in L^{1}(G), f \in A^{1}(G), f(e)=1$. If

$$
\left\{\omega: g^{\wedge}(\omega) \neq 0_{\omega}\right\} \subset\left\{\omega: f^{\wedge}(\omega)=0_{\omega}\right\}
$$

then $g \equiv 0$. Here $0_{\omega}$ is the identically zero transformation on $H_{\omega}$.

This will be a consequence of Theorem 2 below. Given $g \in L^{1}(G)$ we define $g(a)$ to be $g(c a)$. We further define the convolution $f * g$ of two functions $f, g$ by

$$
f * g \cdot(a)=\sum_{b} \tilde{f}(b) g\left(b^{-1} a\right) .
$$

If $f, g \in L^{1}(G)$ then $f * g \in L^{1}(G)$ and $\|f * g\|_{1} \leqslant\|f\|_{1}\|g\|_{1}$; if $f \in L^{1}(G)$ and $g \in L^{2}(G)$ then $f * g \in L^{2}(G)$ and $\|f * g\|_{2} \leqslant\|f\|_{1}\|g\|_{2}$, etc. Moreover if $f \in L^{1}(G)$, and $g, h \in L^{2}(G)$ we have $f *(g * h)=(f * g) * h$. In the present case direct verification of all these formulas is very simple indeed. If

THEOREM 2. Let $G$ be as in (1), let $g \in L^{1}(G), f \in A^{1}(G)$, and let $f(e)=1$.

$$
g *_{c} f \equiv 0
$$

for all $e \leqslant e$ then $g \equiv 0$.
Proof. Our demonstration is an adaptation of an argument taken from Helson ([2], p. 4).

We define $M$ to be the closed linear manifold in $L^{2}(G)$ generated by the functions $\left\{_{c} f\right\}_{c<e}$. We note that for $b$ fixed the mapping $h \rightarrow h(b)$ of $L^{2}(G)$ into the complex numbers satisfies $|h(b)| \leqslant\|h\|_{2}$ and is thus a continuous linear functional on $L^{2}(G)$. Since, if $c<e, c f(a)=0$ for $a \leqslant e$ it follows that if $h \in M$ then $h(a)=0$ for $a \leqslant e$. Now let $f_{M}$ be the projection of $f$ on $M$ and let $k=f-f_{M}$. We have $k(e)=1$ so that $k \neq 0$. Since $k$ is orthogonal to $M$ and since $c^{k \epsilon} \in M$ if $e<e$ we see that

$$
\sum_{a} k(a) \overline{k(c a)}=0 \quad c<e,
$$

which we can rewrite as

$$
k * k^{\sim}(c)=0 \quad \text { for } e>e .
$$

Here $k^{\sim}(a)$ is defined as $\overline{k\left(a^{-1}\right)}$. The identity, $k * k^{\sim} \cdot(o)=\overline{k * k^{\sim}\left(o^{-1}\right)}$, implies that

Finally

Thus

$$
k * k^{2} \cdot(e)=0 \quad \text { for } e<e .
$$

$$
k * k^{\sim} \cdot(e)=\|k\|_{2}^{2} \neq 0 .
$$$$
k * k^{2} \cdot(c)=\delta(c)\|k\|_{2}^{2}
$$

where $\delta(c)$ is 1 if $e=e$ and is 0 otherwise.

It is apparent from (2) that

and thus that

$$
g * k \cdot(a)=0 \text { all } a \in G,
$$

that is, $g \equiv 0$.

$$
\|k\|_{2}^{2} g(a)=g * k * k^{\sim} \cdot(a)=0 \quad \text { all } a \in G ;
$$

Now let $f$ and $g$ satisfy the assumptions of Theorem 1. It is simple to verify and well known that

and

$$
\left({ }_{c} f\right)^{\wedge}(\omega)=U_{\omega}\left(c^{-1}\right) f^{\wedge}(\omega)
$$

$$
\left(g *_{c} f\right)^{\wedge}(\omega)=g^{\wedge}(\omega)[c f]^{\wedge}(\omega)=g^{\wedge}(\omega) U_{\omega}\left(e^{-1}\right) f^{\wedge}(\omega) .
$$

Thus our assumptions imply that for each $\omega \in \Omega$

$$
\left(g *_{c} f\right)^{\wedge}(\omega)=0_{\omega} \quad \text { for all } c \in G .
$$

This in turn, see $[3 ;$ p. 360], implies that

$$
g *_{c} f=0
$$

for all $e \epsilon G$. It follows from Theorem 2 that $g=0$.

An example of a non-commutative group which has an order satisfying (1) is the free group on $n$ letters, $n>1$. See [1] p. 47. 


\section{References}

1]

[1] L. Tuchs

[2] H. Helson, Lectures on Ins, Abstract Harmonic Analysis, Vol. 1., Berlin 1963.

Received June 25, 1971

$(356)$

\section{On the function of Marcinkiewicz}

by

T. WALSH (Princeton N. J.)

Abstract. Define the Marcinkiewicz integral transformation acting on locally integrable functions in $R^{n}$ by

$$
\mu(f)(x)=\left(\left.\left.\int_{0}^{\infty}\left|\int \Omega(y)\right| y\right|^{-n+1} \psi\left(t^{-1} y\right) f(x-y) d y\right|^{2} t^{-3} d t\right)^{1 / 2},
$$

where $\Omega$ is homogeneous of degree 0 . Rearrangement-invariant conditions on $\Omega$ are found under which $\mu$ is bounded in $L^{p}$.

0. Introduction. The Marcinkiewicz function of a locally integrable function of one variable $f$ is defined by

$$
\mu(f)(x)=\left(\int_{0}^{\infty}\left|F^{\prime}(x+t)+F(x-t)-2 F(x)\right|^{2} t^{-3} d t\right)^{1,2},
$$

where $F$ is an indefinite integral of $f$. Stein has considered the following generalization to $n$ variables

$$
\mu(f)(x)=\left(\int_{0}^{\infty}\left|\int_{|y| \leqslant t} \Omega(y) f(x-y) d y\right|^{\mid 2} t^{-3} d t\right)^{1 / 2},
$$

where $\Omega$ denotes a locally integrable function which is homogeneous of degree 0 and has mean value 0 on the unit sphere $S^{n-1}=\{x:|x|=1\}$ with respect to Euclidean surface measure $\sigma$.

Using the boundedness in $L^{p}$ of the 1-dimensional Marcinkiewicz integral transformation Stein showed that if $\Omega$ is odd $\mu$ defined by (1) is also bounded in $L^{p}\left(R^{n}\right)$ for $1<p<\infty$ ([9], Theorem 2). The results for Calderón-Zygmund singular integrals in [4] give rise to the question whether similar results hold for the Marcinkiewicz integral (1) and general kernels.

For a homogeneous function $\Omega$ let $\|\Omega\|_{p}$ denote the $L^{p}$ norm with respect to the measure $\sigma$ on $S^{n-1}$. Also for a positive increasing function $\Phi$ let

$$
\|\Omega\|[\Phi(L)]=\int_{S^{n-1}} \Phi(\mid \Omega(\xi) \|) d \sigma(\xi) .
$$

